Recepción: 17 / 09 / 2016

Aceptación: 31 / $01 / 2017$

Publicación: 29 / 04 / 2017

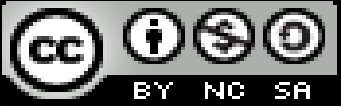

Ciencias económicas y empresariales

Artículo de investigación

\title{
Las nuevas tecnologías de la información en la internacionalización de las empresas
}

The new information technologies in the internationalization of companies Novas tecnologias de informação na internacionalização das empresas

\author{
Jodamia U. Murillo-Rosado \\ jumurillo@sangregorio.edu.ec \\ Luis D. Muñoz-Mendoza"I \\ 1dmunoz@sangregorio.edu.ec \\ Cristian R. Amén-Chinga ${ }^{\text {III }}$ \\ cramen@sangregorio.edu.ec
}

Correspondencia: jumurillo@sangregorio.edu.ec

\footnotetext{
I Magister en Informática Empresarial, Ingeniero en Sistemas Informáticos, Docente de la Universidad San Gregorio de Portoviejo, Portoviejo, Ecuador.

"Magister en Telecomunicaciones, Ingeniero en Sistemas Informáticos, Docente de la Universidad San Gregorio de Portoviejo, Portoviejo, Ecuador.

III Ingeniero Comercial, Docente de la Universidad San Gregorio de Portoviejo, Portoviejo, Ecuador.
} 


\title{
Resumen
}

Con la internacionalización empresarial, la compañía crea las condiciones necesarias para salir al mercado internacional. El proceso de globalización ha hecho que este proceso sea más sencillo gracias a las posibilidades que brindan el internet y el uso de las nuevas tecnologías de la información. Se sabe que las nuevas TIC's ofrecen a las PYMES formas para enfrentar las limitaciones de acceso a los mercados internacionales. Este texto propone reflexionar sobre la importancia del uso de las nuevas tecnologías en el proceso de internacionalización de las empresas, destacando las características particulares que adquieren los procesos empresariales con el uso de las TIC's así como las ventajas e inconvenientes que puede presentar el uso de estos recursos en el ámbito empresarial.

Palabras clave: internacionalización empresarial; TIC; PYMES.

\begin{abstract}
With the internationalization of the company, the company creates the necessary conditions to enter the international market. The process of globalization has made this process simpler thanks to the possibilities offered by the internet and the use of new information technologies. It is known that new ICTs offer SMEs ways to deal with the limitations of access to international markets. This text proposes to reflect on the importance of the use of new technologies in the process of internationalization of companies, highlighting the particular characteristics acquired by business processes with the use of ICT as well as the advantages and disadvantages that can present the use Of these resources in the business field.
\end{abstract}

Keywords: business internationalization; TIC; SMEs.

\section{Resumo}

Com a internacionalização da empresa, a empresa cria as condições necessárias para entrar no mercado internacional. $\mathrm{O}$ processo de globalização tornou este processo mais simples graças às possibilidades oferecidas preferidos pela internet eo uso de novas tecnologias de informação. Sabe-se que as novas TIC oferecem maneiras PME para lidar com as limitações de acesso aos mercados internacionais. Este texto se propõe a refletir sobre a importância do uso de novas tecnologias no processo de internacionalização das empresas, destacando as particularmente 
características adquiridas por processos de negócios com a utilização das TIC, bem como as vantagens e desvantagens que podem apresentar o uso destes recursos na área de negócios.

Palavras chave: internacionalização de negócios; TIC; PME.

\section{Introducción}

La nueva dinámica de la económica mundial y la apertura de economías locales, hace que el mercado ahora sea todo el mundo. Las empresas tienden, cada vez más, a la deslocalización y a dirigir su estrategia hacia el exterior. Este proceso se conoce como internacionalización empresarial y cobra cada vez más importancia a nivel mundial.

La internacionalización empresarial es el proceso por el cual la compañía crea las condiciones necesarias para salir al mercado internacional. Es un proceso a largo plazo, que debe ser organizado en un plan detallado y ejecutado cumpliendo cordialmente con las etapas claves que sean previstas en dicho plan. No se trata de tener una presencia puntual en algún otro país, sino de estar completamente asentado en la economía de esos países.

Para que el proceso logre la calidad requerida hay que tener la estrategia de acción bien definida en el mercado local y entender que los mercados internacionales aportan otras variables a la posibilidad de éxito del producto o servicio: temas culturales, los requisitos sanitarios, ambientales, normas técnicas y reglamentaciones en los productos.

En la actualidad la globalización ha hecho que este proceso sea más sencillo gracias a las posibilidades que brindan el internet y el uso de las nuevas tecnologías de la información. Sin embargo esto no implica que estos recursos sean utilizados de manera arbitraria en el proceso de internacionalización.

En otro orden de análisis, es importante tomar en consideración además que una de las formas de promover el desarrollo, reforzamiento y mejora de la competitividad de las empresas en un país, sobre todo de las PYMES, es a través de la creación de condiciones que les permitan participar en el flujo internacional del comercio. Sin embargo, para crear las condiciones adecuadas para su participación en el contexto actual de una economía mundial globalizada y automatizada, es fundamental que estas empresas estén preparadas para trabajar con los nuevos niveles 
tecnológicos, para poder adoptar los nuevos flujos de información y comunicaciones, y que estos sean dirigidos a lograr el incremento de la productividad y eficiencia empresarial.

La estrategia de internacionalización que una empresa puede implementar conlleva cierta complejidad, dado que las firmas deben evaluar su nivel de preparación para la actividad exportadora, decidir a qué mercado se debe exportar, optando por una estrategia de concentración (uno o pocos países) o de diversificación, buscar información sobre cómo lograr el mejor posicionamiento, realizar viajes para analizar el mercado, promocionar los productos en ferias, adaptar su producto a otros mercados y localizar proveedores y distribuidores, entre otras cuestiones. Sin embargo, en el marco del proceso de globalización y creciente apertura de los mercados, se convierte en una estrategia cada vez más necesaria.

Es cada vez más evidente que las nuevas TIC's ofrecen a las PYMES la posibilidad de enfrentar las limitaciones de acceso a los mercados internacionales. El presente trabajo tiene como propósito reflexionar sobre la importancia del uso de las nuevas tecnologías en el proceso de internacionalización de las empresas, destacando las características particulares que adquieren los procesos empresariales con el uso de las TIC's, así como las ventajas e inconvenientes que puede presentar el uso de estos recursos en el ámbito empresarial.

\section{Desarrollo}

Bajo el enfoque estratégico, la internacionalización de empresas es el resultado de la adopción de una serie de estrategias en las que se consideran tanto los recursos y capacidades de la empresa como las oportunidades y amenazas del entorno (Araya, 2009: 18). Este resulta un proceso de gran importancia que permite a las empresas posicionarse en el mercado internacional y proyectar sus actividades, total o parcialmente, a un entorno internacional. Lo anterior favorece a la empresa en tanto propicia que se generen flujos de diversos tipos como: comerciales, financieros y de conocimiento entre distintos países.

Según Canals (1994) los objetivos de internacionalizar una empresa son:

- Apertura de nuevos mercados.

- Costes de producción más bajos. 
- Una estructura de la producción y la distribución de la empresa más eficiente.

El proceso de internacionalización en el caso de las PYMES requiere optimizar todos los recursos que están a su disposición, en este sentido, la utilización intensiva de las Tics, es uno de los recursos claves para el logro de este cometido. Mientras mayor sea el aprovechamiento de este recurso por parte de las empresas, mayor será el impacto en el grado de internacionalización de las mismas (Fernández, 2002).

La adopción de las Tics por parte de las PYMES puede pensarse no solo como un reto, sino más bien como una importante oportunidad para los países denominados "en vías de desarrollo", debido a las implicaciones sobre la capacidad de este sector de generar mayores oportunidades de empleo y riqueza. Puede considerarse como la más importante implicación de la adopción de las Tics por parte de estas empresas, sea la posibilidad de desarrollar nuevas ventajas comparativas basadas en la información y la creatividad para desarrollar nuevos productos y servicios, lo cual contrasta con la necesidad de poseer mucho capital, mano de obra o recursos naturales para poder generar tales.

\section{Ventajas}

El uso de las TIC's por las PYMES puede acelerar el desarrollo y las capacidades de internacionalización de estas empresas, siempre que sepan adaptarlas a sus necesidades reales. Otro elemento a tener en cuenta son las ventajas y desventajas que puede tener el uso de estos recursos tecnológicos, de forma tal que en las empresas puedan amortiguarse los inconvenientes que puedan generarse como resultado de la introducción de las nuevas tecnologías en el escenario empresarial.

Las ventajas que ofrece el uso de las TIC`s a las PYMES pueden verse en tres direcciones fundamentales:

\section{Difusión de la información dentro de la empresa:}

Esto se realiza con el objetivo de brindar y compartir la información mediante el uso del correo electrónico y las páginas web. En la actualidad esto ocurre no sólo en las grandes empresas globales, multinacionales o transnacionales, sino que se ha extendido hacia las PYMES aunque 
no en igual medida. Aun cuando existen diferencias notables en el acceso y uso que hacen las PYMES del internet en comparación con las grandes empresas, este se ha constituido en uno de los recursos más utilizados en el ámbito empresarial.

La adopción de internet puede tener utilidad directa en varios aspectos que facilitan la internacionalización: la búsqueda de información de la industria, de los competidores y del mercado potencial a nivel global; la investigación sobre nuevos productos y nuevos proveedores, encontrar recursos o información básica para nuevos negocios. A su vez, la implementación de internet permite utilizar otras herramientas como buscadores, grupos de discusión, listas de correo electrónico, boletines, etc.

Internet, al ser un servicio relativamente simple ofrecido por sitios web públicos y privados, permite a los usuarios registrados presentar y transferir automáticamente solicitudes y ofertas comerciales e información, para brindar oportunidades de hacer el primer contacto con potenciales compradores y proveedores a nivel global.

Otro aspecto significativo es que las PYMES pueden apoyarse en los mensajes de correo electrónico y otros métodos de telecomunicación como los sistemas de videoconferencia, y utilizarlos en forma complementaria para iniciar acuerdos.

2. Mejora de la gestión interna de la empresa:

En este sentido las empresas desarrollan e introducen aplicaciones para lograr mayor eficiencia y racionalización del trabajo, entre otras cosas la contabilidad, la planificación comercial, la administración del personal, la planificación y el control de producción y la gestión del inventario.

En la actualidad, una alternativa recurrente para que las pequeñas y medianas empresas puedan aumentar sus oportunidades de exportación, es que participen en cadenas de suministro organizadas por empresas grandes. Para poder aprovechar estas oportunidades, las Pymes invierten en Tics para fortalecer y aumentar la eficiencia de sus procesos internos de concepción, desarrollo de productos, producción, gestión de calidad y otras actividades administrativas.

3. Intercambio de información con clientes y proveedores: 
Este es el uso más avanzado de las Tics, mediante el cual las empresas buscan desarrollar vínculos de colaboración con otras empresas y clientes mediante la conexión de redes. Se comparte información con proveedores y clientes, y se busca desarrollar un papel de colaboración con empresas asociadas, con el objeto de aumentar la eficiencia en la gestión de la producción, la gestión del inventario, las ventas y la distribución, o para desarrollar la gestión de la cadena de suministro.

Este tipo de aplicación de las TIC`s permite a la empresa identificar los productos y servicios que requieren sus clientes, optimizar los canales de entrega, identificar grupos de clientes para desarrollar estrategias, obtener conocimientos acerca de las necesidades actuales de sus clientes, entender a los grupos dominantes de clientes para establecer relaciones a largo plazo, aumentar las ventas junto con la satisfacción del cliente.

Esta es una manera global de gestionar las relaciones que la empresa tiene con sus clientes, incluidos los clientes potenciales, para obtener ventajas mutuas de larga duración. Permite además que las empresas adapten sus estrategias comerciales y productivas a las necesidades de los clientes en cada región.

El uso de TIC`s por las PYMES y su constante perfeccionamiento, le proporciona a estas empresas una mayor posibilidad de presencia internacional, incrementando su potencial de internacionalización.

Uno de los medios fundamentales de los que puede auxiliarse las PYMES para el proceso de internacionalización es la aplicación del comercio electrónico, del que puede obtener como resultado nuevas formas de competir en el mercado internacional, donde se difuminan las ventajas de que goza la gran empresa cuando actúa sobre otros medios.

El comercio electrónico puede utilizarse en cualquier entorno en el que se intercambien documentos entre empresas: compras o adquisiciones, finanzas, industria, transporte, salud, legislación y recolección de ingresos o impuestos. Se utiliza fundamentalmente para desarrollar los siguientes aspectos:

- Creación de canales nuevos de marketing y ventas. 
- Acceso interactivo a catálogos de productos, listas de precios y folletos publicitarios.

- Venta directa e interactiva de productos a los clientes.

- Soporte técnico ininterrumpido, permitiendo que los clientes encuentren por sí mismos, y fácilmente, respuestas a sus problemas mediante la obtención de los archivos y programas necesarios para resolverlos.

Mediante el comercio electrónico se intercambian los documentos de las actividades empresariales entre socios comerciales. Los beneficios que se obtienen en ello son: reducción del trabajo administrativo, transacciones comerciales más rápidas y precisas, acceso más fácil y rápido a la información, y reducción de la necesidad de reescribir la información en los sistemas de información.

Los tipos de actividad empresarial que podrían beneficiarse mayormente de la incorporación del comercio electrónico son:

- Sistemas de reservas: centenares de agencias dispersas utilizan una base de datos compartida para acordar transacciones.

- Stocks: aceleración a nivel mundial de los contactos entre proveedores de stock.

- Elaboración de pedidos: posibilidad de referencia a distancia o verificación por parte de una entidad neutral.

- Seguros: facilita la captura de datos.

- Empresas proveedoras de materia prima a fabricantes: ahorro de grandes cantidades de tiempo al comunicar y presentar inmediatamente la información que intercambian.

Además de lo señalado anteriormente, uno de los principales beneficios del comercio electrónico específicamente para las PYMES, es la posibilidad de llegar a nuevos mercados y clientes así como la flexibilidad y adaptabilidad que permite en los negocios y la rapidez en las transacciones. Supone una gran oportunidad de mercado, ya que permite ampliar el horario de 
venta las 24 horas del día y que cualquier cliente potencial pueda acceder a su tienda independientemente del lugar del mundo en que se encuentre.

Las principales características que tiene el comercio electrónico y que les permite a las PYMES acelerar el proceso de internacionalización son:

1.- Presencialidad: en el comercio tradicional, un mercado es un lugar físico que se visita para realizar transacciones, con el comercio electrónico, el producto que ofrece la empresa está disponible en cualquier parte y en todo momento.

Este tipo de comercio permite comprar desde el escritorio del cliente, su hogar, su oficina y distintos lugares. Esto permite que se reduzcan los costos de la transacciones: costos por participar en un mercado y también reduce la energía cognoscitiva que define el esfuerzo mental requerido para completar una tarea.

2.- Alcance: la tecnología del comercio electrónico permite que las transacciones comerciales traspasen los límites culturales y nacionales con mucha mayor conveniencia y efectividad en costos de lo que se puede lograr con el comercio tradicional. El total de usuarios o clientes que un negocio de comercio electrónico puede obtener es una medida de su alcance.

3.- Amplios estándares: los estándares técnicos universales de internet y del comercio electrónico reducen considerablemente los costos de entrada al mercado: esto se evidencia en el costo que deben pagar los comerciantes solo por llevar sus bienes al mercado; para los consumidores estos estándares reducen los costos de búsqueda lo que se expresa en el esfuerzo requerido para encontrar el producto adecuado, y el descubrimiento de precio es más simple.

4.- Mejora de la información: esto se refiere a la complejidad y contenido de un mensaje, internet tiene la facilidad y el potencial para ofrecer mucha más riqueza que el comercio tradicional, gracias a que es interactiva y puede ajustar el mensaje a cada usuario individual; la riqueza del servicio web permite a los comerciantes de ventas de bienes y de servicios, comercializar productos que hasta ahora requerían de presentación directa. 
5.- Interactividad: es la tecnología que permite la comunicación de dos vías entre el comerciante y el cliente, la interactividad permite que un comerciante en línea se comprometa con un cliente en formas similares a una experiencia cara a cara, pero a una escala global mucho más masiva.

6.- Incremento de la información: es el monto y la cantidad total de información disponible para todos los participantes en el mercado, clientes y comerciantes por igual; la información se vuelve más plena, menos costosa y de mayor calidad.

7.- Adecuación y personalización: la personalización es la capacidad de dirigir mensajes de marketing a individuos específicos, ajustando el mensaje al nombre de una persona, sus intereses y sus compras anteriores.

La adecuación se refiere a la capacidad de cambiar el producto o servicio entregado con base en las preferencias o el comportamiento anterior de un usuario. Esta permite que las firmas identifiquen con precisión los segmentos de mercado y que ajusten sus mensajes de acuerdo con sus objetivos.

8.- Tecnología social: permite que los usuarios creen y compartan contenido en forma de texto, videos, música o fotos con una comunidad mundial. Internet proporciona un modelo de muchos a muchos de las comunicaciones masivas que es único.

Estas características que presenta el comercio electrónico, evidencian que su uso adecuado y efectivo puede incidir de manera favorable en la internacionalización de las empresas, sobre todo de las PYMES. Este recurso permite a las PYMES tener acceso a nuevos clientes y expandir geográficamente sus mercados. La tecnología de la información en sentido general, tiene el inmenso poder de reducir los costos de coordinación, comunicación y procesamiento de información sobre los mercados, clientes, etc. Esto crea nuevas oportunidades para las PYMES en cuanto a la posibilidad de comercializar sus productos en el mercado exterior.

Sin embargo hay que tener en cuenta que las TIC no generan de por sí, competitividad en la empresa, sino que para poder incentivarla su incorporación y extensión debe ser acompañada por cambios profundos, no sólo a nivel técnico sino también organizacional, en la relaciones con proveedores, clientes, etc., que permita la generación y circulación de conocimiento, y una estructura organizativa que facilite la comunicación y el aprendizaje. 
Lo anterior requiere entonces que las PYMES tengan en cuenta las ventajas y desventajas que tiene el uso de las nuevas tecnologías, no sólo para la propia empresa sino también para los usuarios, pues esto permitirá que las estrategias vayan encaminadas a disminuir los riesgos e inconvenientes que representa el uso de las TIC's por las PYMES y potenciar las ventajas que trae consigo.

Las ventajas que ofrece para las empresas son:

- Elimina obligaciones con trabajadores por los contratos.

- Disminuye los costos de los distribuidores.

- Elimina las perdidas por mercancía robada.

- Genera mayores ganancias por venta unitaria de un producto.

- Desaparición de los límites geográficos y temporales.

- Incremento de los índices de ganancia en concepto de ventas, pues estas se pueden realizar 24 horas al día durante los 365 días del año.

- Reducción de costes tanto de producción, administración y almacenamiento.

- Obtención de un mayor número de clientes tanto online como offline gracias al aumento de visibilidad que permite internet.

- Flexibilidad en los métodos de pago.

- Facilidad para ofrecer una comparativa entre productos, incluyendo características y precios.

Las principales desventajas son:

- Disminuye la comunicación entre vendedor y consumidor.

- Peligro con la presencia de hackers que pueden perjudicar los ingresos de la empresa.

- Inseguridad con respecto al método de pago y la calidad del producto. 
- Intangibilidad, la imposibilidad de poder tocar y comprobar la calidad suele tirar hacia atrás a algunos clientes.

- Distancia entre el cliente y el vendedor.

- Los gastos de envío.

- Gastos de promoción.

En relación a los usuarios las principales ventajas que tiene este tipo de comercio son:

- Posibilidad de encontrar un producto a menor costo.

- Realizar mejor negociación con el vendedor.

- Comodidad en la adquisición del bien o producto.

Entre las desventajas se pueden destacar:

- Dificultad para proceder con una queja del producto.

- Cobro o poder hacer válida la garantía del producto comercializado.

- Se pierde la capacidad de visualización del producto en comercialización o conocimiento físico del producto.

\section{Conclusiones}

La internacionalización se ha convertido en una necesidad para la supervivencia de las empresas, sobre todo para las PYMES, pues el nuevo entorno internacional requiere que las empresas desarrollen capacidades dinámicas que les permita competir en los mercados actuales y futuros.

Sin embargo esto no es un proceso natural, las ventajas que se logran de la internacionalización de la empresa, se derivan esencialmente de la capacidad que tiene cada una para coordinar actividades de la cadena de valor agregado. Este proceso permite a las PYMES poder subsistir ante la globalización y la alta competencia en un mercado cada vez más complejo. 
Los procedimientos de entrada en los mercados internacionales pueden ser muy variados: la exportación, la inversión directa o la concesión de licencias. Para cualquiera de estos procedimientos las empresas pueden aprovechar las oportunidades que brindan las TIC's y como la adecuada implementación de estas puede lograr perfeccionar el proceso de internacionalización.

\section{Referencias bibliográficas}

Eclac (2007). "Using ICT and trade facilitation tools for SME development - options for latin america and the caribbean", FAL bulletin (facilitation of trade and transport in latin america and the caribbean), United Nations.

Haynes, P. J., Becherer, R. C., and Helms, M. M. (1998). "Small and mid-sized businesses and internet use: unrealized potential?" Internet research: electronic networking applications and policy, 8(3), 229-235.

Neef, Dale (editor) (1998). The knowledge economy: resources for the knowledge-based economy. EEUU: butterworth \& heinemann.

Araya Leandro, Arnoldo. (2009). El proceso de internacionalización de las empresas. Revista TEC empresarial, Vol. 3, Ed 3.

Canals, J. (1994). La internacionalización de la empresa. Madrid: McGraw-Hill.

Fernández, Z. y Nieto, M.J. (2002). "La estrategia de internacionalización de la pequeña empresa familiar". Documento de trabajo 02-18 (100). Series de economía de la empresa. 